\title{
Evaluación del comportamiento frente a la corrosión de recubrimientos procesados con láser de alta potencia
}

\author{
G.P. Rodríguez*, M. Checa*y J.J. de Damborenea**
}

\begin{abstract}
Resumen Los aceros inoxidables presentan altas resistencias a la corrosión pero cuando trabajan en determinados medios a elevadas temperaturas aparecen problemas de oxidación. Una solución a este problema consiste en aplicar recubrimientos utilizando láseres de alta potencia. Los recubrimientos objeto de estudio en este proyecto han sido procesados con un láser de $\mathrm{CO}_{2}$ a partir de polvos compuestos de $\mathrm{NiCrAl}$ y $\mathrm{ZrO}_{2} / \mathrm{Y}_{2} \mathrm{O}_{3}$. Se ha estudiado la influencia de las condiciones de procesado de los recubrimientos en la calidad de los cordones, microestructura obtenida, resistencia a la oxidación y resistencia a la corrosión electroquímica. Las condiciones de procesado estudiadas han sido la velocidad de desplazamiento del láser respecto de la muestra y el caudal de los polvos formadores del recubrimiento.
\end{abstract}

Palabras clave Recubrimientos láser. Corrosión electroquímica. Oxidación. Aceros inoxidables.

\section{Corrosion behaviour of high-power laser coatings}

\begin{abstract}
Stainless steels present high corrosion resistance but oxidation problems can appear at high temperature. Possible solutions could be the application of coatings by high power laser. Laser-cladding experiments have been performed with $\mathrm{NiCrAl}-\mathrm{ZrO}_{2} / \mathrm{Y}_{2} \mathrm{O}_{3}$ powder on stainless steel substrates. The influence of the powder feed rate and the laser scanning speed has been investigated. The best processing conditions have been determined by evaluating microstructure, high temperature oxidation resistance and corrosion resistance.
\end{abstract}

Keywords

Laser coatings. Electrochemical corrosion. Oxidation. Stainless steel.

\section{INTRODUCCIÓN}

En las últimas décadas, se ha producido un avance espectacular en el desarrollo de nuevos materiales capaces de trabajar bajo condiciones cada vez más agresivas. La investigación en la utilización del láser ha permitido obtener sobre los materiales metálicos nuevas superficies más resistentes al desgaste, corrosión y oxidación a elevadas temperaturas mediante tratamiento térmico, aleación superficial o procesado de recubrimientos ${ }^{[1}$ y 2$]$. Además, se ha demostrado su importancia en el mantenimiento y reparación de componentes usados o desgastados.

Los recubrimientos con $\mathrm{NiCrAl}$ son apropiados para aquellas partes o piezas expuestas a medios agresivos a elevadas temperaturas debido a sus altas resistencias a la corrosión y a la oxidación. Los recubri- mientos, además de tener elevados puntos de fusión se oxidan lentamente dando lugar a capas protectoras formadas fundamentalmente por $\mathrm{Al}_{2} \mathrm{O}_{3}{ }^{[3]}$. Asimismo, ante un proceso de corrosión electroquímica, se desarrollan capas pasivas que protegen al material. La presencia de aluminio permite la formación de compuestos intermetálicos del sistema $\mathrm{Ni}$ - $\mathrm{Al}$ que endurecen el recubrimiento ${ }^{[4]}$. La adición de $\mathrm{ZrO}_{2}$ y $\mathrm{Y}_{2} \mathrm{O}_{3}$, dispersos en los polvos de partida, mejoran la adherencia y estabilizan las capas de óxidos formados frenando la degradación de la aleación ${ }^{[5]}$.

\section{MATERIALES Y PROCEDIMIENTO EXPERIMEN-} TAL

El material base consiste en un acero inoxidable austenítico AISI316L y los polvos de partida en una 
mezcla de $\mathrm{NiCrAl}$ y $\mathrm{ZrO}_{2} / \mathrm{Y}_{2} \mathrm{O}_{3}$ con la siguiente composición: $66 \mathrm{Ni}-16 \mathrm{Cr}-12 \mathrm{Al}-3 \mathrm{Zr}-1 \mathrm{Y}$ (\% peso). Los recubrimientos se procesan con un láser de $\mathrm{CO}_{2}$ continuo con una potencia de salida máxima de 5 $\mathrm{kW}$ mediante la técnica de inyección de polvos. Con el fin de evitar la oxidación, el procesado se realiza en atmósfera de argón suministrado con un caudal de $45 \mathrm{l} / \mathrm{min}$. Los recubrimientos se obtienen bajo diferentes condiciones de caudal de polvos: 74 , 85 y $93 \mathrm{~g} / \mathrm{l}$ y velocidades de barrido del láser: 500, $700,1.000$ y $1.200 \mathrm{~mm} / \mathrm{min}$. Sobre cada probeta se realizan 6 cordones de $6 \mathrm{~mm}$ de anchura (Fig. 1).

Se estudia la influencia de las variables de procesado en la microestructura del cordón y en la naturaleza de las fases presentes mediante microscopía óptica, electrónica de barrido (SEM) y espectroscopia de energías dispersas de RX (EDX). Asimismo se estudia la resistencia a la corrosión electroquímica en medio salino $(\mathrm{NaCl} \mathrm{0,06} \mathrm{M)}$ utilizando técnicas potenciostáticas y analizando las curvas de polarización obtenidas, en cada caso.

Para caracterizar el comportamiento frente a la oxidación de los cordones se introducen en un horno previamente calentado a $1.000{ }^{\circ} \mathrm{C}$, durante períodos de tiempo variables ( $24 \mathrm{~h}, 96 \mathrm{~h}$ y $168 \mathrm{~h}$ ) $y$, posteriormente, se enfrían en aire. Se estudia la microestructura y aspecto de los óxidos con ayuda de un microscopio óptico y electrónico; se analiza la composición química de las distintas fases del cordón producidas por el tratamiento térmico, así como los óxidos formados.

\section{RESULTADOS Y DISCUSIÓN}

Los cordones procesados con láser presentan, en general, buen aspecto, si bien un estudio más detallado indica que los cordones procesados con la

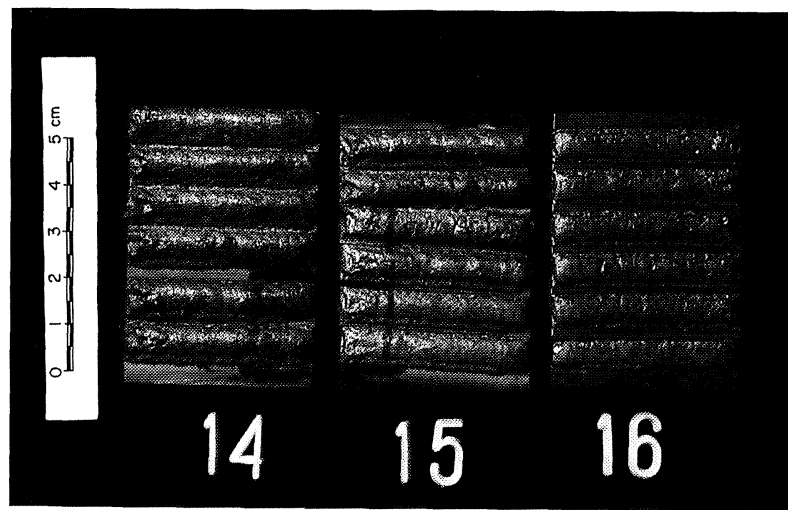

Figura 1. Aspecto de los cordones procesados con láser.

Figure 1. Appearance de of the laser tracks. menor velocidad de desplazamiento del haz láser (700 $\mathrm{mm} \mathrm{min}^{-1}$ ) y mayor caudal $\left(93 \mathrm{~g} \mathrm{~min}^{-1}\right)$, presentan microrrechupes o porosidades en su interior. Este efecto no se aprecia en el resto de condiciones de procesado. Cuando las probetas se procesan con el mismo caudal $\left(73 \mathrm{~g} \mathrm{~min}^{-1}\right)$ se observa una influencia de la velocidad de procesado: a medida que aumenta la velocidad de desplazamiento del láser aumenta la presencia de microgrietas en el cordón (en algunos casos van desde la interfase hasta la superficie del recubrimiento).

El tamaño del cordón es función de la velocidad de desplazamiento del láser y del caudal de polvos. En las condiciones ensayadas ha variado entre 1.050 y $2.350 \mathrm{~m}$ el espesor y entre $6.100 \mathrm{~m}$ y $7.000 \mathrm{~mm}$ la anchura.

La microestructura formada presenta una interfase entre el recubrimiento y el material base, caracterizada por ser una zona continua sin imperfecciones o heterogeneidades, que permite asegurar una buena unión metalúrgica entre el sustrato y el material de aporte. Desde la interfase aparece una zona estrecha de crecimiento no epitaxial de granos columnares y, a continuación (ocupando casi la totalidad del cordón), una zona de microestructura dendrítica (Fig. 2).

$\mathrm{El}$ aspecto de las dendritas es diferente dependiendo de las distintas velocidades de desplazamiento del láser; al aumentar la velocidad de procesado la distancia entre espaciados interdendríticos es menor (Fig. 3). Asimismo, la velocidad de desplazamiento del láser influye en la dirección del crecimiento de las dendritas. A mayor velocidad se produce un mayor cambio en la orientación, lo que confiere un carácter más anisótropo al

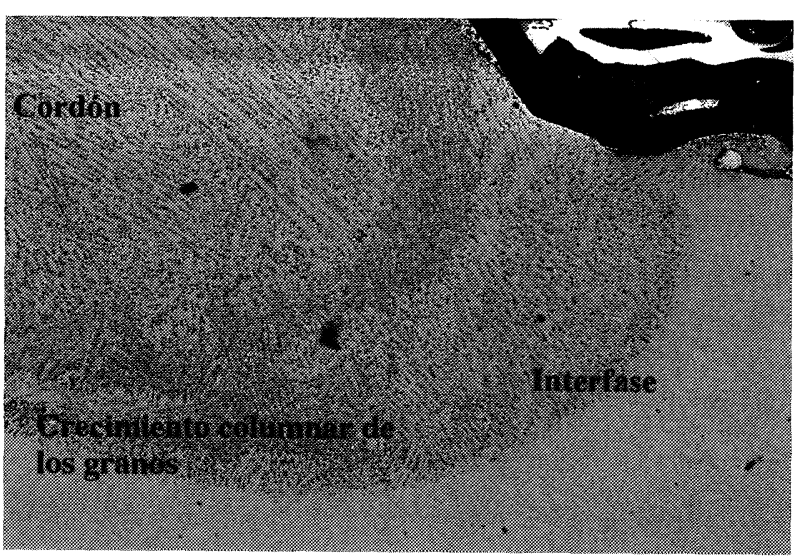

Figura 2. Microestructura del cordón procesado a 1.000 $\mathrm{mm} \mathrm{min}^{-1}, 74 \mathrm{~g} \mathrm{~min}^{-1}$ (x 125).

Figure 2. Microstructure of the track obtained at 1,000 mm $\mathrm{min}^{-1}, 74 \mathrm{~g} \mathrm{~min}^{-1}$ (x 125).

Rev. Metal. Madrid Vol. Extr. (2005) 513-517 

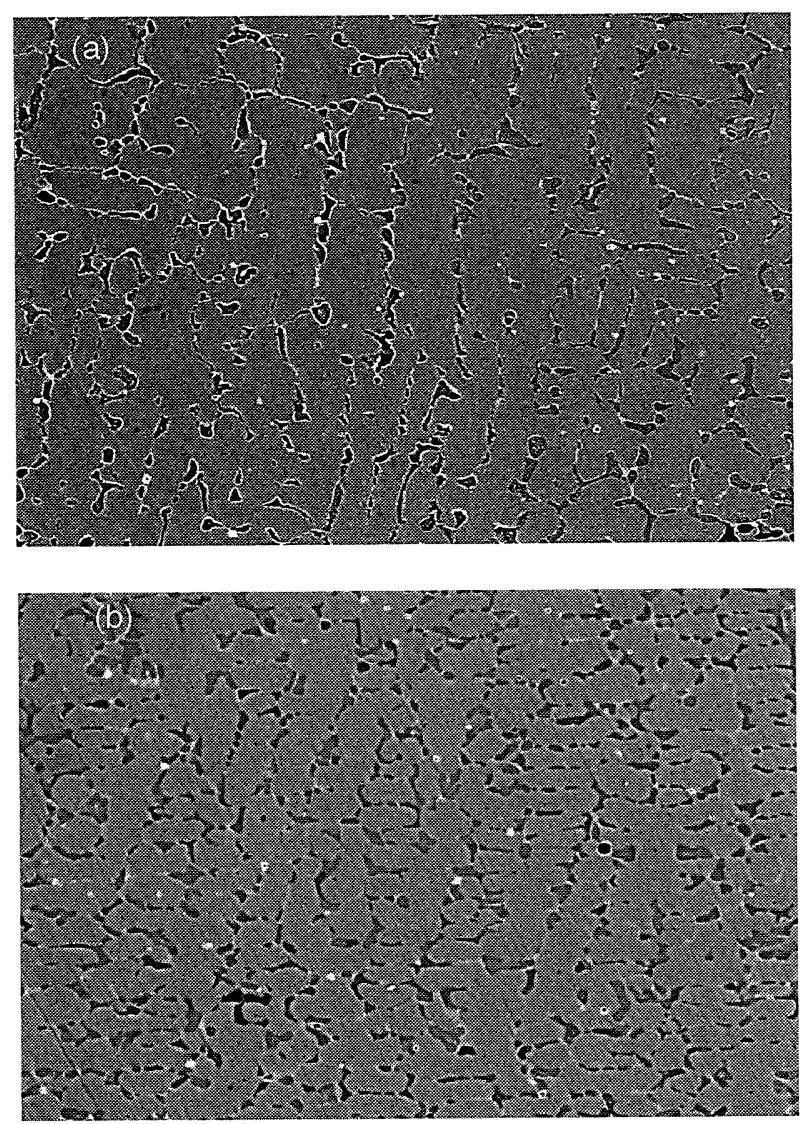

Figura 3. Microestructura del cordón procesado (a) $700 \mathrm{~mm} \cdot \mathrm{min}^{-1}$ y (b) $1.000 \mathrm{~mm} \cdot \mathrm{min}^{-1}$ (x 1.000).

Figure 3. Microstructure of the track obtained at (a) $700 \mathrm{~mm} \cdot \mathrm{min}^{-1}$ and (b) 1,000 $\mathrm{mm} \cdot \mathrm{min}^{-1}$ (x 1,000).

cordón (Fig. 4). La anisotropía presentada por la estructura dendrítica generada durante los procesos de fusión y solidificación es un aspecto a tener en cuenta para evaluar las mejores condiciones de procesado.

En la figura 5 se presenta con mayor detalle la microestructura y las fases analizadas. La fase 1 representa el centro de la dendrita, la cual está formada mayoritariamente por una solución sólida de níquel con cromo, hierro y aluminio. La fase 2 representa los espacios interdendríticos que se corresponden con compuestos intermetálicos enriquecidos en aluminio del tipo $\mathrm{Ni}_{3} \mathrm{Al}$; en el que parte del nitrógeno ha sido desplazado por el cromo y el hierro, formándose durante la solidificación fases metaestables con la estequiometría $(\mathrm{NiCrFe})_{3} \mathrm{Al}$. Alrededor de esta última fase, se aprecia una tercera (partículas blancas), que corresponde a la precipitación las partículas cerámicas $\left(\mathrm{ZrO}_{2}\right)$.

Los análisis muestran como en el recubrimiento aparece hierro (no presente en los polvos de parti-
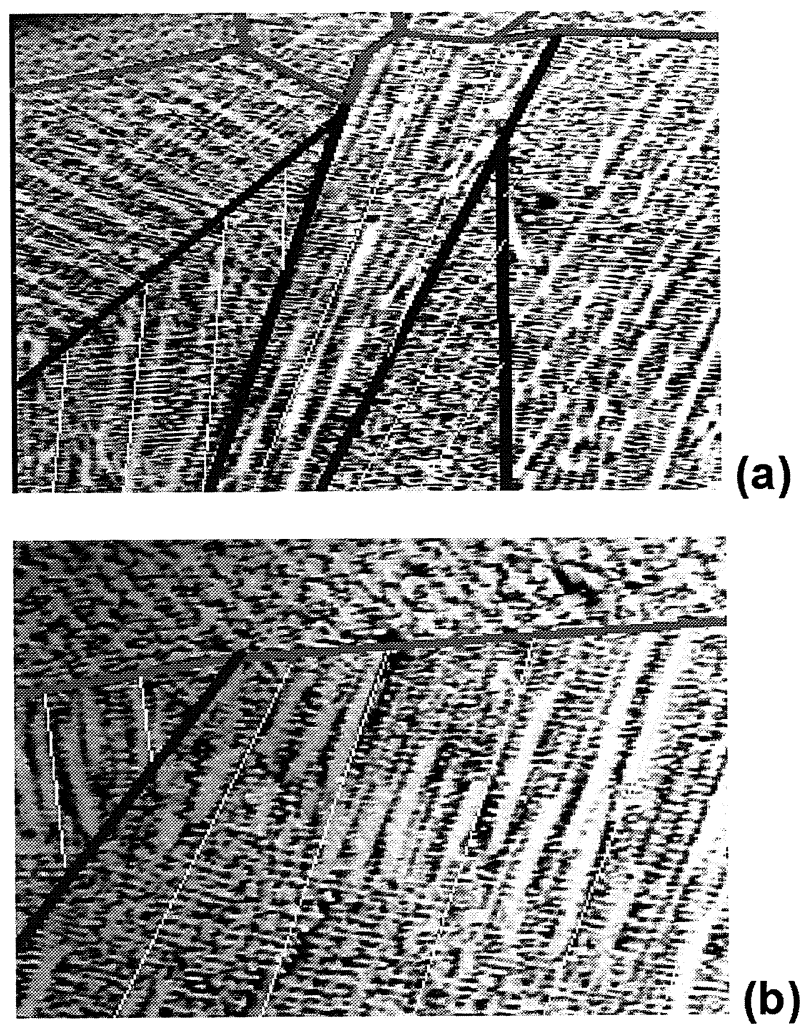

Figura 4. Cambio de orientación en el crecimiento dendrítico cerca de la superficie del cordón, a) $1.000 \mathrm{~mm} \cdot \mathrm{min}^{-1}$, b) $700 \mathrm{~mm} \cdot \mathrm{min}^{-1}$ ( $\left.\times 50\right)$.

Figure 4. Change in the dendritic growth direction near the surface a) $1,000 \mathrm{~mm} \cdot \mathrm{min}^{-1}$, b) $700 \mathrm{~mm} \cdot \mathrm{min}^{-1}(x$ 50).

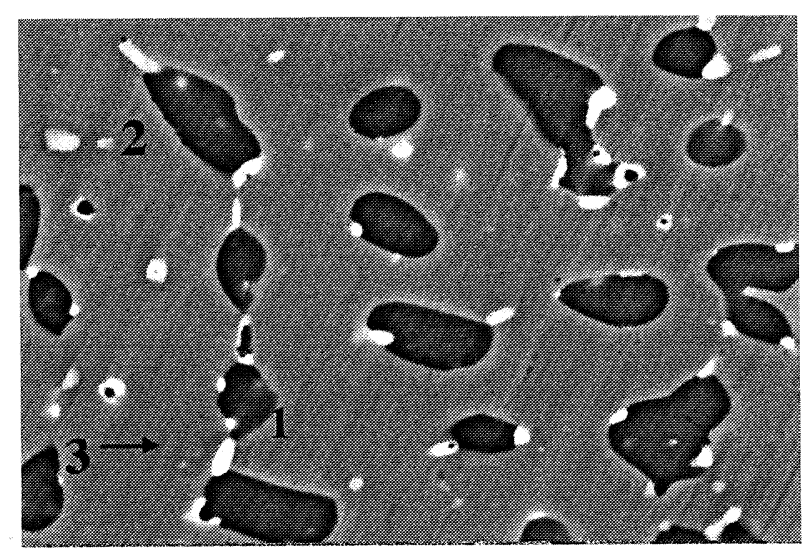

Figura 5. Microestructura del cordón (x 4.000).

Figure 5. SEM micrograph of the laser track $(x 4,000)$.

da), debido a que se produce difusión de este elemento desde el material base al cordón. Este enriquecimiento en hierro es mayor en los cordones procesados a menor velocidad, ya que la temperatura y el tiempo de calentamiento han sido mayores.

Las curvas de polarización registradas durante los ensayos de corrosión electroquímica indican 
que las mejores condiciones de procesado son las correspondientes a $700 \mathrm{~mm} \mathrm{~min}^{-1}$ y $73 \mathrm{~g} \mathrm{~min}^{-1}$ (menor velocidad y menor caudal de alimentación). Esto, es debido a dos razones: por un lado, bajo estas condiciones, el recubrimiento presenta una mayor composición en aluminio y cromo (elementos pasivantes) y, por otro lado, el cordón es más homogéneo (libre de grietas, rechupes, irregularidades en el aspecto final de los cordones). En la figura 6 se presentan las curvas de polarización correspondientes a dos cordones procesados a distintas velocidades, $700 \mathrm{~mm} \mathrm{~min}^{-1}$ y $1.000 \mathrm{~mm} \mathrm{~min}^{-1}$ para un caudal constante de alimentación de $74 \mathrm{~g} \mathrm{~min}^{-1}$.

Cuando el material base se introduce a $1000^{\circ} \mathrm{C}$ en atmósfera de aire sufre un proceso de oxidación, que ya es significativo para un tiempo de exposición de 24 h. Los efectos devastadores del proceso de oxidación sobre el material base justifican la necesidad de procesar cordones sobre la superficie del material, que permitan reducir este efecto.

En general, la oxidación del material recubierto conduce a la formación de una estrecha capa de óxido exterior homogénea sobre toda la superficie, sin apreciarse propagación del ataque hacía el interior del cordón. Los óxidos formados son, mayoritariamente, óxidos de aluminio y níquel, pero a medida que aumenta el tiempo de oxidación se reduce el óxido de níquel formado para aumentar la cantidad de óxido de aluminio, presentando un valor de un $81 \%$ at. Al para un tiempo de $168 \mathrm{~h}$.

También, la microestructura del recubrimiento se ve afectada por el tratamiento a $1.000^{\circ} \mathrm{C}$. Se ha observado, cómo en las zonas más superficiales sólo aparece la fase 1, desplazándose la fase 2 hacia el interior. Además, al aumentar el tiempo de oxidación se produce una disminución del contenido del aluminio alcanzándose, incluso, valores tan bajos que para tiempos de $168 \mathrm{~h}$ no se detecta en la superficie

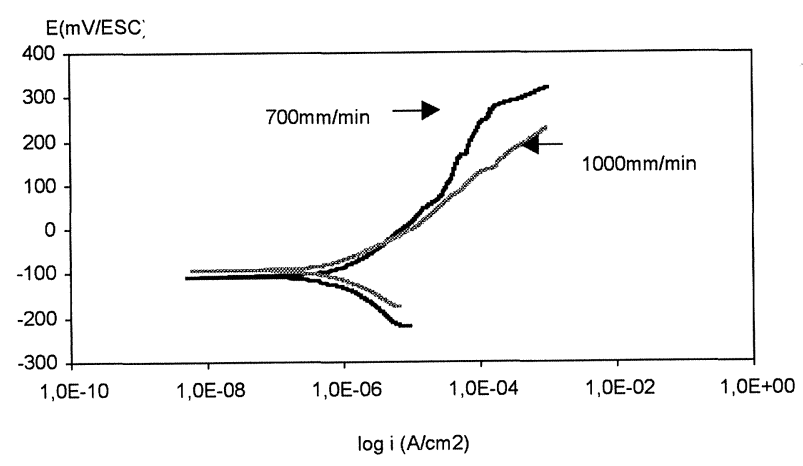

Figura 6. Curvas de polarización de cordones procesados con láser.

Figure 6. Polarization curves for the laser tracks. del recubrimiento. Asimismo, al aumentar el tiempo del tratamiento térmico, se produce una transformación de la estructura dendrítica del recubrimiento para formar una estructura globular (Fig. 7)

Para velocidades de procesado de $1.000 \mathrm{~mm}$ $\min ^{-1}$ y superiores, el proceso de oxidación se produce de forma heterogénea no afectando por igual a toda la superficie, apreciándose en muchos casos, propagación de óxido hacia el interior del cordón.

\section{CONCLUSIONES}

Los recubrimientos compuestos de $\mathrm{NiCrAl}$ con $\mathrm{ZrO}_{2}-\mathrm{Y}_{2} \mathrm{O}_{3}$, procesados con un láser de alta potencia, incrementan la resistencia a la oxidación a $1.000{ }^{\circ} \mathrm{C}$ de los aceros inoxidables. Existe una influencia importante de las condiciones de procesado del recubrimiento en su comportamiento frente a la oxidación y a la corrosión electroquímica. Se puede concluir que las mejores condiciones de procesado han sido las correspondientes a una velocidad de desplazamiento del láser de $700 \mathrm{~mm} \mathrm{~min}^{-1} \mathrm{y}$

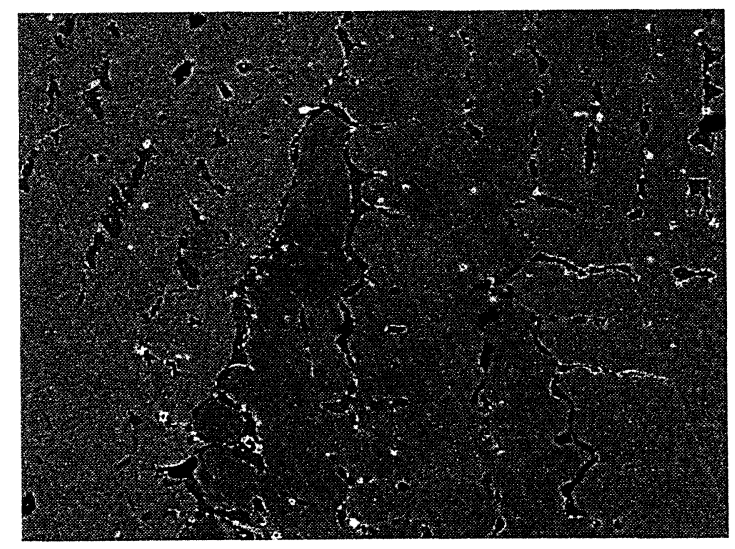

(a)

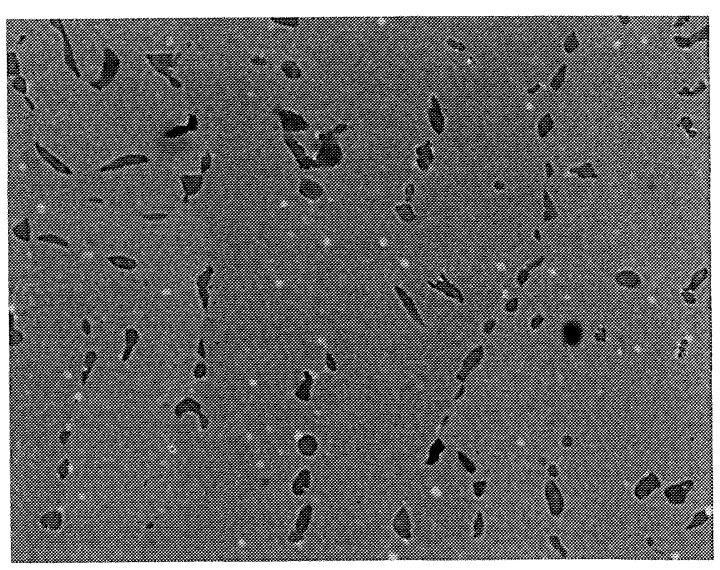

(b)

Figura 7. Microestructura del cordón tras un proceso de oxidación (a) 24 h, (b) 168 h (x 1.000).

Figure 7. Microstructure of the laser track after oxidation (a) $24 h$, (b) $168 h(x 1,000)$.

Rev. Metal. Madrid Vol. Extr. (2005) 513-517 
Evaluación del comportamiento frente a la corrosión de recubrimientos procesados con láser de alta potencia G.P. RodRíGuez, M. CHECA Y J.J. DE DAMBORENEA

un caudal de polvos de $73 \mathrm{~g} \mathrm{~min}^{-1}$ (menor velocidad y menor caudal de alimentación).

\section{REFERENCIAS}

[1] G. Múñiz, A.L. Conde, B.J. Fernández, R. Varela, I. García, J.J. de Damborenea, Rev. Metal. Madrid 39 (2003) 443-451.
[2] J. De Damborenea, Surf. Coat. Technol. 100-101 (1998) 377-382.

[3] D. Schlager, C. Theiler y H. Kohn, Mater. Corros. 53 (2002) 103-110.

[4] M. SCHNEIDER, Tesis Doctoral, University of Twente, Enschede, Netherlands, 1998.

[5] B.A. PINT, Oxid. Met. 49 (1998) 531-559. 


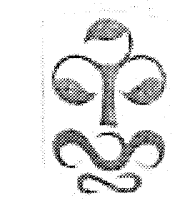

UnW

bes
VEO

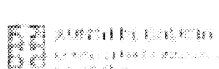
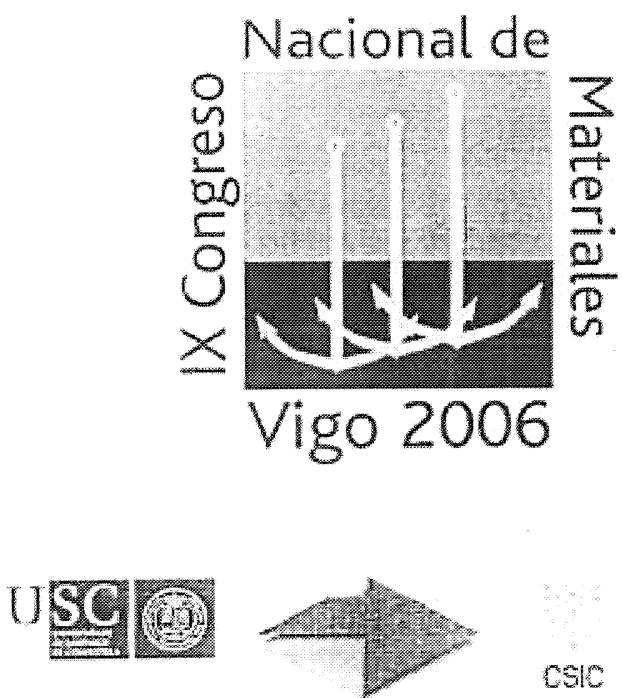

$\operatorname{csic}$

AIMEN
3

官

S E (iii) A T

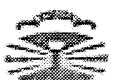

caxamoma? 


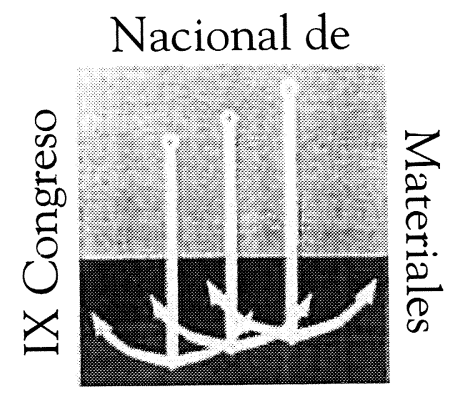

\section{Congreso Nacional de Materiales Vigo 20, 21 y 22 de junio de 2006}

Vigo 2006

\section{ÁREAS TEMÁTICAS:}

- Materiales metálicos.

- Materiales poliméricos.

- Materiales cerámicos.

- Recubrimientos y superficies (capas delgadas, tribología, etc.)

- Materiales compuestos.

- Corrosión, degradación y protección.

- Comportamiento mecánico y fractura.

- Cementos, hormigones y otros materiales de construcción.

- Materiales funcionales (Semiconductores, superconductores, materiales magnéticos, fotónicos, etc.)

- Biomateriales.

- Nanomateriales.

\section{FECHA CLAVE}

- Recepción resúmenes: 25 de Noviembre de 2005

- Aceptación de resúmenes: 7 de Febrero de 2006

- Recepción trabajos: 7 de Abril de 2006

- Fecha límite de inscripción: 7 de Abril de 2006 (tarifa reducida)

\section{SECRETARÍA DEL CONGRESO}

Área de Ciencia de Materiales.

Escuela Técnica Superior de Ingenieros Industriales.

c/ Maxwell, 9

Universidad de Vigo.

Campus Lagoas-Marcosende

C.P.: 36310 , Vigo

\section{Telfs: 986812603}

986812229

E-mail: ixcnm@uvigo.es 


\section{PRESENTACIÓN DE TRABAJOS A REVISTA DE METALURGIA}

\section{INSTRUCCIONES TÉCNICAS PARA LOS AUTORES}

Revista de Metalurgia es una publicación de ámbito internacional sobre ciencia y tecnología de materiales metálicos, cuyo principal objetivo es contribuir a la difusión de los nuevos conocimientos científicos y desarrollos tecnológicos que se producen en este campo. En ella, se recogen principalmente trabajos sobre los procedimientos de obtención, fabricación, transformación y reciclado de metales y aleaciones, las propiedades y características tecnológicas de los mismos, así como las diferentes aplicaciones de estos materiales.

Revista de Metalurgia es una publicación del Centro Nacional de Investigaciones Metalúrgicas, perteneciente al Consejo Superior de Investigaciones Científicas.

\section{TIPO DE TRABAJOS}

1.1. Originales. Contribuciones en las que se describan y discutan los resultados de trabajos de I+D o de experiencias industriales de interés.

1.2. Revisiones. Revisiones bibliográficas o críticas sobre el conocimiento actual en campos específicos de ciencia y tecnología metalúrgicas.

1.3. Notas técnicas. Trabajos en los que se describan experiencias prácticas sobre procedimientos de laboratorio y de taller, técnicas de ensayo y caracterización, comportamiento en servicio de los materiales, aplicaciones industriales, etc.

1.4. Comunicaciones. Contribuciones breves en las que se informe o se adelanten resultados.

\section{PRESENTACIÓN DE LOS ORIGINALES}

\subsection{Envío de los trabajos}

\subsubsection{Envío postal}

Los trabajos se enviarán a la Redacción en soporte papel (copia impresa), acompañados de las figuras originales y una copia de las mismas, además de una copia en un disquete de 3,5" (formateado para $\mathrm{PC}$ ) o un CD-ROM, conteniendo todo el trabajo.

La dirección correcta para los envíos es:

Revista de Metalurgia
Redacción. CENIM
Avda. de Gregorio del Amo, 8
28040 Madrid (España)

Los trabajos irán acompañados de una carta original en la que se solicitará la publicación del trabajo y se indicará la dirección completa del autor al que debe dirigirse toda correspondencia relacionada con el mismo.

\subsubsection{Envío electrónico}

También se admitirán trabajos remitidos por correo electrónico, que siempre deberán incluirse en un fichero adjunto al mensaje de presentación de los mismos. En este caso, en el transcurso del proceso de edición, el autor deberá hacer llegar a la Redacción, en soporte papel, la carta original firmada en la que solicita la publicación del trabajo, así como las fotografías, figuras e ilustraciones originales y una copia de las mismas.

La dirección electrónica es:

\section{revista@cenim.csic.es}

\subsection{Originalidad, lenguaje y estilo}

Se presentarán exclusivamente trabajos originales que no hayan sido editados anteriormente en ningún tipo de publicación.

Aunque los trabajos deberán redactarse en español o en inglés, el título, que debe reflejar el contenido del trabajo, debe redactarse en ambos idiomas. A continuación, debe figurar el nombre completo de cada uno de los autores del trabajo seguido del primer apellido (en el caso de desear que figuren dos apellidos, separarlos por un guión).

El resumen debe redactarse también en español y en inglés y debe describir el contenido del trabajo en 150 palabras como máximo. Al final del resumen se incluirán cinco palabras clave, en español y en inglés.

No debe duplicarse la presentación de datos mediante su inserción en figuras y tablas.

El texto del trabajo debe dividirse en secciones y subsecciones numeradas. Las páginas deben numerarse correlativamente.

Las referencias, ecuaciones, tablas y figuras deben numerarse correlativamente según su inserción en el texto (las referencias entre corchetes [1], [2], [3-6]..., las ecuaciones entre paréntesis, como (1), (2), (3)..., las tablas con números romanos, como I, II, III...y las figuras como Fig. 1, Fig. 2, Fig. 3...).

\subsection{Presentación de trabajos}

\subsubsection{En soporte papel}

La extensión del texto escrito de los trabajos originales remitidos no superará las 25 páginas a doble espacio, excluido el espacio ocupado por tablas y figuras.

Además del texto, debe aparecer en páginas separadas cada uno de los apartados siguientes:

a) El título en español y en inglés, seguido por los nombres y las direcciones de los autores.

b) El resumen y las palabras clave (en español y en inglés).

c) Las referencias bibliográficas.

d) Cada una de las figuras.

e) La relación de todos los pies de las figuras (en español y en inglés).

f) Cada una las tablas con su correspondiente cabecera en español y en inglés.

g) Los posibles agradecimientos.

\subsubsection{En soporte informático}

Es obligatorio enviar una copia electrónica que contenga el fichero del texto del trabajo original, en un disquete de ordenador PC $\left(3,5^{\prime \prime}\right)$, un CD-ROM o por correo electrónico. Además, deben adjuntarse, si es posible, los ficheros que correspondan a las figuras, fotografías e ilustraciones que lo acompañan. Los ficheros de texto corresponderán a procesadores de uso universal (preferentemente Word 97) y serán remitidos en formato de texto (.doc), a ser posible no comprimido. Para los ficheros gráficos o de imagen se indicará el programa con que pueden abrirse: En todo caso, en el mensaje de presentación del trabajo, debe especificarse claramente el formato de cada uno de los ficheros de texto o gráficos, el tipo de fichero, la extensión de los mismos y los programas con que están creados y modificados. Para más detalles, los autores pueden dirigirse a la Redacción de la Revista.

\section{4. Referencias bibliográficas}

Las referencias bibliográficas aparecerán insertadas en el texto en orden correlativo, con el número correspondiente escrito entre corchetes y, preferentemente, como superíndice $\left({ }^{[l}\right)$ ya que así serán publicadas. Al final del trabajo, en una hoja separada, deben relacionarse todas, correlativamente, con los datos y la puntuación que se indica a continuación:

\subsubsection{Criterios generales}

Todos los datos de las referencias son obligatorios. En los parágrafos siguientes se indican cuáles son aquellos, según el tipo de publicación. Los ejemplos muestran el formato y las características tipográficas que deben aplicarse a cada dato y a la notación de separación entre los mismos. Es recomendable que las referencias incluidas en los trabajos se expresen del mismo modo.

No deben incluirse referencias a comunicaciones confidenciales o a publicaciones restringidas que no sean fácilmente accesibles para los lectores. 
En el caso de que deban figurar dos apellidos de un autor, se indicarán separándolos por un guión.

Deben indicarse, habitualmente, los números de página inicial y final y separados por un guión. No se aceptarán referencias en las que no se incluya, al menos, el número de página inicial.

Las abreviaturas de los títulos de las revistas deben ajustarse a las incluidas en la norma UNE-1008-77 (ISO 4-1972). A título orientativo, puede consultarse la guía Bibliographic Guide for Editors $\mathcal{E}$ Authors, que publica la American Chemical Society.

\subsubsection{Publicaciones periódicas}

Inicial(es) del nombre de cada autor seguido del primer apellido, título abreviado de la revista, volumen, número de fascículo o mes (si las páginas de los diferentes fascículos anuales no son continuadas), año de publicación, número de las páginas inicial y final.

\subsubsection{Libros o monografías}

Inicial(es) del nombre de cada autor seguido del primer apellido, título del libro, nombre de la editorial, número de edición, ciudad de la edición, país, año de publicación, número de las páginas inicial y final.

\subsubsection{Resúmenes (Actas, Proceedings) de congresos o conferencias}

Inicial(es) del nombre de cada autor seguido del primer apellido, título del congreso, volumen de los resúmenes, ciudad de la celebración, país, año de la celebración, nombre del editor(es), nombre de la editorial, ciudad de la edición, país, año de publicación, número de las páginas inicial y final.

\subsubsection{Tesis Doctorales}

Inicial(es) del nombre del autor seguido del primer apellido, Facultad, Universidad, año de calificación de la Tesis.

\subsubsection{Ejemplos}

[1] C. CORNEJO y J. GARCÍA-NÚÑEZ, Rev. Metal. Madrid 34 (1998) 235-239.

[2] F. R. MORRAL, E. JIMENO y P. MOLERA, Metalurgia General, Ed. Reverté, Barcelona, España, 1985, pp. 161-168.

[3] J. G. SMITH y R. H. JONES, Proc. ICSMA-8, vol. 2, Tampere, Finlandia, 1987, T. Thomas y R. Wilson (Eds.), Pergamon Press, Oxford, Inglaterra, 1988, pp. 131-136.

[4] L. ASENJO, Tesis Doctoral, Facultad de Ciencias Físicas, Universidad Complutense de Madrid, 1995.

\subsection{Ilustraciones, figuras y micrografías}

\subsubsection{Presentación}

Cada figura o ilustración debe presentarse en una hoja separada. Los pies de las mismas, redactados en español y en inglés, deben permitir una correcta interpretación de aquellas sin que sea preciso acudir al texto. Además, los pies de todas las figuras que aparezcan en el trabajo se relacionarán conjuntamente en hoja aparte y correlativamente. Las figuras deben ser de una calidad que permita una buena impresión. Es obligatorio enviar un juego de figuras originales $\underline{\square}$ una copia del mismo que se procurará sean de buena calidad para su correcta impresión. Las figuras que incluyen gráficos, en los que aparezcan ejes cartesianos no deben presentarse con trama de fondo, cuadrícula o sub-ejes, a no ser que sea imprescindible para interpretar la figura.

\subsubsection{Micrografías y fotografías}

Las micrografías, y en algunos casos las fotografías, deben especificar los aumentos por medio de una escala en el interior de las mismas. En casos excepcionales se podrá indicar los aumentos en el pie de figura, como en el ejemplo siguiente: " $\times 150$ ". Además, en el reverso de las mismas y escrito a lápiz, debe hacerse constar el nombre del autor y el número de figura.

\subsubsection{Color}

Las figuras, micrografías o fotografías serán editadas en blanco y negro aunque el original enviado sea de color. Sólo se publicarán en color cuando el autor, expresamente, acepte abonar los gastos extra ocasionados.

\subsubsection{Tamaño y colocación}

Las figuras se enviarán de un tamaño igual o mayor al de las dimensiones finales a las que aparecerá reproducido en la Revista. Estas dimensiones son: ancho de columna $(80 \mathrm{~mm})$ o ancho de página $(170 \mathrm{~mm})$. La altura final de las leyendas y expresiones de las figuras, tras la reducción correspondiente, debe ser de un mínimo de $3 \mathrm{~mm}$.

Cuando una figura esté formada por dos o más ilustraciones o micrografías relacionadas entre sí, de modo que todas las ilustraciones se correspondan con el mismo número de figura, se indicará cuál es la a), b), c),... mediante la inserción de la letra correspondiente en la figura.

\subsection{Tablas}

Las tablas deben llevar un encabezamiento, en español y en inglés, y deben ser inteligibles sin necesidad de acudir al texto. Las tablas no presentarán trama alguna y las divisiones horizontales y verticales de los datos se limitarán a las imprescindibles para la comprensión de los mismos.

\subsection{Unidades}

Tanto en el texto como en las ilustraciones sólo se utilizarán los símbolos y abreviaturas del Sistema Internacional de Unidades (SI). Como excepción se admitirá que las temperaturas se expresen en grados centígrados y los tiempos largos en horas.

\subsection{Pruebas de imprenta}

El autor con el que se mantenga la correspondencia sobre el trabajo recibirá, con carácter general, un juego de pruebas de imprenta para su verificación. En todo caso, se le solicitará la conformidad al texto final que haya sido preparado con las correcciones de los revisores o de la Redacción de la Revista antes de su envío a la imprenta. En esta etapa de la edición del trabajo no se admitirá la inclusión de material nuevo.

\subsection{Separatas y ejemplares impresos}

$\mathrm{Al}$ autor con el que se mantenga la correspondencia sobre el trabajo se le enviará tantos ejemplares del número de la Revista donde aparezca aquel como autores tenga el trabajo. Se pueden enviar separatas, a cargo de los autores y a los precios de costo de las mismas, siempre y cuando se haga constar al devolver, una vez corregidas, las pruebas de imprenta del trabajo.

\subsection{Devolución de fotografías y originales}

En el caso de rechazarse expresamente la publicación de un trabajo se devolverá el material original aportado a la Revista. Cuando el trabajo no llegara a publicarse por haber sido retirado por los autores en cualquiera de sus fases previas a la publicación, el material original sólo se devolverá por petición expresa recibida en los 30 días siguientes a la retirada.

\section{DERECHOS DE AUTOR}

$\mathrm{Al}$ remitir un trabajo, los autores acceden a que sus derechos sobre el mismo sean transferidos al editor siempre y cuando el mencionado trabajo sea aceptado para su publicación. Estos derechos de autor cubren los derechos en exclusiva para reproducir y distribuir el artículo en cualquier soporte y las traducciones del mismo. Con carácter general, no se devolverán a los autores ni los originales en soporte papel e informático, ni las figuras o ilustraciones originales correspondientes a los trabajos publicados. 


\section{PRESENTATION OF PAPERS TO REVISTA DE METALURGIA}

\section{TECHNICAL INSTRUCTIONS FOR AUTHORS}

Revista de Metalurgia is a journal with an international scope dealing with metals materials science and technology, the main objective of which is to contribute to the dissemination of new scientific discoveries and technological development in this field. The journal mainly gathers work on the process of obtaining, producing, transforming and recycling metals and alloys, their properties and technological characteristics and the various applications of those materials.

Revista de Metalurgia is a publication of the Centro Nacional de Investigaciones Metalúrgicas belonging to the Consejo Superior de Investigaciones Científicas.

\section{TYPE OF PAPERS}

1.1. Originals. Contributions in which the results of the $R+D$ work or industrial experiments of interest are described and discussed.

1.2. Reviews. Bibliographic or critical reviews of the current knowledge in specialised scientific fields and metallurgic technology.

1.3. Technical notes. Work which describes practical experience about laboratory and workshop processes, experiments and characterisation techniques, service behaviour of materials, industrial applications, etc.

1.4. Communications. Brief contributions that inform or anticipate results.

\section{PRESENTATION OF ORIGINAL DOCUMENTS}

\subsection{Sending the work}

\subsubsection{By post}

The work should be sent to the editorial office in paper form (printed copy), together with the original diagrams and copies, as well as a copy on a 3.5 "diskette (formatted for PCs) or a CDROM, containing all the work.

The work should be sent to:

\section{Revista de Metalurgia \\ Redacción. CENIM \\ Avda. de Gregorio del Amo, 8 \\ 28040 Madrid (España)}

The work should be accompanied by an original letter in which the publication of the work will be requested and which states the author's complete correspondence address.

\subsubsection{By e-mail}

Work sent by e-mail will also be accepted if it is in a file attached to an introductory message. In this case, the author, during the course of the editing process, will have to send an original signed letter, on paper, to the editorial office in which he requests the publication of the work, together with the photographs, diagrams and original illustrations, and a copy of each.

The e-mail address is:

\section{revista@cenim.csic.es}

\subsection{Originality, language and style}

Only original work, which has never been published before in any kind of publication, should be presented.

Although the work will be written in either Spanish or in English, the title, which must reflect the content of the work, has to be written in both languages. Then, the complete first name followed by the first surname of each of the authors, (if you wish two surnames to appear, separate them with a hyphen).
The summary has also to be written in Spanish and English and must describe the content of the work in 150 words maximum. Five key words in Spanish and English should be included at the end of the summary.

Data presented in either figures or tables should not be duplicated in the second format.

The text has to be divided in numbered sections and subsections. The pages must be numbered.

The references, equations, tables and diagrams must be numbered according to their insertion in the text (references between square brackets [1], [2], [3-6]..., equations between brackets (1), (2), (3)..., tables with Roman numerals I, II, III... and diagrams as Fig. 1, Fig. 2, Fig. 3...).

\subsection{Presentation of the work}

\subsubsection{On paper}

The length of the original written texts should not exceed 25 pages, double-spaced, excluding the space used for tables and diagrams.

As well as the text, each of the following headings must appear on separate pages:

a) The title in Spanish and English, followed by the names and addresses of the authors.

b) The summary and key words (in Spanish and English).

c) The bibliographical references.

d) All of the figures.

e) A list of all the figure captions (in Spanish and English).

f) Each of the tables with its corresponding heading in Spanish and English.

g) Any acknowledgements.

\subsubsection{On disk}

It is compulsory to send an electronic copy that contains the original text file, on a 3.5" computer diskette, a CD-rom or by email. The files that correspond to the diagrams, photographs and illustrations that accompany the text should also be attached, if possible.

The text files should correspond to universal processors (preferably Word 97) and be sent in text format (.doc), not compressed if possible. With graphic or image files the program with which it can be opened should be indicated. In all cases, the cover letter of the work should clearly specify the format of each of the text or graphic files, the type of file, their length, and the programs with which they were created and modified. For more details, authors can contact the magazine's editorial staff.

\subsection{Bibliographical references}

The bibliographical references should appear in the text in correlative order, with the corresponding number written in brackets and preferably as superscript $\left({ }^{[}\right)$, as they will be published that way. At the end of the work, on a separate piece of paper, the list of references should be listed, correlatively, with the information and punctuation that is listed below:

\subsubsection{General criteria}

All the information about the references is obligatory. Listed in the following paragraphs are those necessary, according to the type of publication. The examples show the format and the typographic characteristics that should be applied to the information and the separation notation between them. It is recommended that the references included in the works are expressed in the same way.

References to confidential communications or to restricted publications should not be included if they are not easily accessible for the readers.

In the case that two surnames of an author should appear, they should be separated by a hyphen. 
It is customary to indicate the first and last page numbers of the reference and separate them with a hyphen. References will not be accepted that do not include, at least, the first page number.

The abbreviations of journal titles should abide by those included in the rule UNE- 1008-77 (ISO 4-1972). For guidance, you can consult the Bibliographic Guide for Editors $\mathcal{E}$ Authors, which is published by the American Chemical Society.

\subsubsection{Newspaper publications}

The initial(s) of each authors' name followed by the first surname, abbreviated journal title, volume, instalment number or month (if the pages of the different annual instalments are not continuous), year of publication, first and last page numbers.

\subsubsection{Books or monographs}

The initial(s) of each authors' name followed by the first surname, book title, editorial number, edition number, publishing city, country, year of publication, first and last page numbers.

\subsubsection{Congress or conference summaries (Minutes, Proceedings)}

The initial(s) of each authors' name followed by the first surname, congress title, volume number of the minutes, host city, country, year held, editors' names, editorial name, publishing city, country, year of publication, first and last page numbers.

\subsubsection{Doctorate Theses}

The initial(s) of the author followed by the first surname, Faculty, University, year of thesis qualification.

\subsubsection{Examples}

[1] C. CORNEJO y J. GARCÍA-NÚÑ̃EZ, Rev. Metal. Madrid 34 (1998) 235-239.

[2] F. R. MORRAL, E. JIMENO y P. MOLERA, Metalurgia General, Ed. Reverté, Barcelona, España, 1985, pp. 161-168.

[3] J. G. SMITH y R. H. JONES, Proc. ICSMA-8, vol. 2, Tampere, Finlandia, 1987, T. Thomas y R. Wilson (Eds.), Pergamon Press, Oxford, Inglaterra, 1988, pp. 131-136.

[4] L. ASENJO, Tesis Doctoral, Facultad de Ciencias Físicas, Universidad Complutense de Madrid, 1995.

\subsection{Illustrations, diagrams and micrographs}

\subsubsection{Presentation}

Each diagram or illustration must be presented on a separate piece of paper. Their figure captions, written in Spanish and English, should allow their correct interpretation without having to consult the text. The figure captions of all the diagrams that appear in the work should also be listed together, correlatively, on a separate piece of paper. The figures must be of a quality that allows good reproduction. It is obligatory to send a set of original figures and copies that will secure that they are of good enough quality to be reproduced correctly. Figures that include graphics in which appear Cartesian axes should not be presented with shaded backgrounds, grids or sub-axes, whenever they are not necessary in order to interpret the diagram.

\subsubsection{Micrographs and photographs}

Micrographs, and in some cases photographs, should specify the enlargements on a scale placed inside them. In exceptional cases the enlargements can be indicated at the base of the figure, as given in the following example "x 150". On the back of the micrographs and written in pencil the author's name and the figure number should also be stated.

\subsubsection{Colour}

Diagrams, micrographs or photographs will be printed in black and white even though the original may be colour. Colour photographs will only be published when the author expressly accepts to pay for the extra costs incurred.

\subsubsection{Size and placing}

The figures should be sent equal to or larger than the dimensions it will actually appear printed in the journal. These dimensions are: column width $(80 \mathrm{~mm})$ or page width $(170 \mathrm{~mm})$. The final height of the figures headings and expressions, after the corresponding reduction, must be a minimum of $3 \mathrm{~mm}$.

When a diagram is composed of two or more related illustrations or micrographs, meaning that all the illustrations correspond to the same figure number, they should be indicated as a), b), c)... by inserting the corresponding letter in the figure.

\subsection{Tables}

The tables should have a heading, in Spanish and English, and should be understandable without reference to the text. The tables should not have any shading and the horizontal and vertical lines of the information should be limited to only those necessary for their comprehension.

\subsection{Units}

In the text, as in the illustrations, only symbols and abbreviations from the International Units System (SI) should be used. The use of temperature expressed in degrees centigrade and long periods of time expressed in hours will be allowed as exceptions.

\subsection{Printing proofs}

Authors that stay in contact with us about their work will receive, as a general rule, a set of printing proofs for verification. In all cases, approval of the final text that has been prepared with the proof readers' or magazine editors' corrections will be sought before being sent to print. At this stage of the work's publication inclusion of new material will not be permitted.

\subsection{Offprints and printing examples}

Authors that stay in contact with us about their work will be sent one example of the journal edition where their work appears per author of the work. Offprints can be sent, in the charge of the authors and at cost prices, whenever the return of the works' corrected print proofs is certified.

\subsection{Return of photographs and originals}

Should the publication of a piece of work be expressly rejected the original material sent to the journal will be returned. When the work is not published due to withdrawal by the authors at any stage before publication, the original material will only be returned by express request received within 30 days following the withdrawal.

\section{AUTHOR'S RIGHTS}

By sending the work the authors agree that their rights to the same are transferred to the editor whenever the work is accepted for publication. These include the exclusive rights to reproduce and distribute the article in whatever format and includes translations of the work. Generally, the original work on paper and diskette, the diagrams and the original illustrations corresponding to the published work will not be returned to the authors. 